\title{
Prácticas de crianza en la sala cuna y en el hogar: un estudio de caso*
}

\author{
ASTRID BRAVO SOTO** \\ Universidad Católica de la Santísima Concepción - Chile
}

Recibido el 19-01-2017; primera evaluación el 27-06-2017; segunda evaluación el 01-03-2018; tercera evaluación el 18-05-2018; aceptado el 28-05-2018

\section{Resumen}

El ingreso de los bebés a espacios educativos formales los instala en un contexto distinto a su hogar, debido a la presencia de otras personas, elementos y dinámicas. El artículo presenta parte de los hallazgos de una investigación que explora los intercambios en torno a la crianza de bebés de cinco a catorce meses entre las familias y un centro educativo. Se describen las prácticas de crianza de los diferentes contextos, los intercambios que se generan en torno a estas y su introducción en el currículum. Se observa que las prácticas en la sala cuna se orientan desde un enfoque de derechos, enfatizan actividades con fines pedagógicos y fomentan un buen trato hacia el bebé. En el contexto familiar, se da preferencia al contacto afectivo con los bebés. La inclusión de saberes sobre la crianza en la propuesta curricular es aún incipiente.

Palabras clave: crianza, educación inicial, currículum

\footnotetext{
* La investigación fue financiada con fondos del Concurso Interno de Proyectos de Investigación de la Universidad Católica de la Santísima Concepción, Chile.

** Educadora de párvulos, magíster en Educación, mención Curriculum de la Universidad de Concepción de Chile. Actualmente, se desempeña en el Departamento de Curriculum y Evaluación de la Facultad de Educación de la Universidad Católica de la Santísima Concepción. Imparte docencia en las asignaturas de Teorías Curriculares y Curriculum de la Educación Parvularia Primer y Segundo Ciclo. Su línea de investigación y sus publicaciones se vinculan con el ámbito de la educación de niños y niñas de cero a tres años. Teléfono de contacto: 56-41- 2345391. Correo: abravo@ucsc.cl
} 


\title{
Child rearing practices at infant toddler center and at home: a case study
}

\begin{abstract}
Infant entrance to formal education spaces installs them in a different context, with new people, things and dynamic. The article reports part of a study who explores the exchange about child rearing practices between families and a day care center. Child rearing practices of different contexts, the exchanges that take place about this and how is gathered in the curriculum are described. It is noted that infant - toddler classroom practices are based on rights approach, activities are emphasized by pedagogic purposes and a good treatment to the child is improved. In the familiar context, affective contact with infants is prioritized. The incorporation of knowledge concerning rearing practices in the proposal curriculum is still incipient.
\end{abstract}

Keywords: child rearing, initial education, curriculum

\section{Práticas de criaçáo na creche e na casa: um estudo do caso}

\section{Resumo}

A inserção de bebês em contextos educacionais formais instala os em outro contexto com novas pessoas, elementos e dinâmicas. $\mathrm{O}$ artigo a presenta parte de um estudo que explora o intercâmbio em torno de criação dos bebês entre famílias e um centro educacional. Descreve as características das práticas de criação num contexto educativo formal e num familiar, intercâmbio que se produz ao redor destas e como são recolhidas estes saberes na proposta curricular. Observa se que na creche práticas eles são orientados a partir do enfoque de direitos, enfatizam-se atividades com fins pedagógicos e um bom trato para a criança. No contexto familiar, se prioriza o contato afetivo com os bebês. A inclusão de saberes sobre criação na proposta curricular é ainda incipiente.

Palavras-chave: criação, educação infantil, currículo 


\section{INTRODUCCIÓN}

La preocupación por el desarrollo y educación de párvulos de cero a tres años se ha incrementado durante las últimas décadas. Influenciada, tanto por el aporte de múltiples disciplinas como por la Convención de los Derechos del Niño, esta se ha ido plasmando en distintas legislaciones, políticas y programas. En esta línea, Chile ha creado un Sistema de Atención Integral a la Infancia y ha ampliado la cobertura de atención en el nivel sala cuna, entre otras medidas. La última Encuesta de Caracterización Socioeconómica Nacional indica que solo un $29,1 \%$ de párvulos de cero a tres ańos asiste a un centro educativo (Ministerio de Desarrollo Social, 2016).

El currículum prescrito para la educación parvularia señala a la familia como el principal agente educativo y al centro educativo como una instancia de apoyo y acompañamiento. Subraya la importancia de las comunidades de pertenencia de los párvulos, enfatizando en el respeto y valoración en cuanto a su diversidad étnica, lingüística y cultural; asimismo, promueve una construcción curricular que recoja saberes provenientes de los contextos más próximos al párvulo (Ministerio de Educación, 2005).

La especificidad del nivel educativo que acoge a niños y nińas, en sus primeros años de vida, su función, las características que adquiere la enseńanza, así como lo que constituye contenido son cuestiones que pertenecen a un campo teórico aún en ciernes (Marotta, 2009; Violante, 2008a). La tendencia de la política del nivel a incorporar una mayor cantidad de bebés a contextos educativos formales y las definiciones curriculares que apuntan a una educación compartida con la familia exigen investigar el ámbito de la construcción curricular en este ciclo educativo.

El escrito expone referentes del problema que se aborda. Muestra las principales características de la crianza en un contexto educativo formal, así como lo que describen las madres como sus prácticas en el hogar. Señala, además, características del intercambio que se genera en torno a la crianza y su incorporación en la propuesta curricular del centro. Por último, discute algunos hallazgos.

\section{La CONSTRUCCIÓN CURRICULAR EN LA SALA DE LOS BEBÉS}

En Chile, la incorporación de párvulos a establecimientos educativos comienza durante los primeros meses de vida, específicamente, al nivel sala cuna, el cual los acoge hasta los dos años. Los bebés están a cargo de un profesional, educador(a) de párvulos, quien se encarga de diseńar, implementar 
y evaluar el currículo, así como de promover un trabajo articulado con la familia y comunidad para favorecer aprendizajes en los párvulos, acorde con las bases curriculares elaboradas por el Ministerio de Educación. Dentro de los lineamientos establecidos en este referente curricular se incluyen los criterios de participación y de contextualización en el proceso de planificación, y el fomento de una construcción curricular que recoja saberes provenientes de las familias y de las comunidades donde se desarrolla la acción educativa (Ministerio de Educación, 2005).

El currículum se relaciona con la selección y transmisión de saberes dentro del contexto escolar. La incorporación de bebés a contextos educativos formales implica pensar en las propuestas curriculares que se generan para estos, lo que comporta una reflexión acerca de lo que se selecciona como contenido a enseñar en este nivel educativo, así como sus referentes.

Educar bebés resulta una tarea distinta a la de otros niveles educativos, «más que en cualquier otra sala, estos necesitan contacto afectivo, corporal y la resignificación de las actividades cotidianas» (Pitluk, 2011, p. 129). De igual modo, Ramírez (2009) subraya que lo que se enseña a los bebés es más que lo prescrito en el currículum, también incluye lo que se hace por medio de actitudes personales, la distribución del tiempo y del espacio, los mensajes que se dan en la cotidianeidad y que no necesariamente están declarados en el currículo oficial. En consecuencia, las dinámicas diarias en el centro educativo son las que imprimen el carácter pedagógico por medio de la forma que los adultos tienen de acoger al bebé, de hablarle, de ofrecerle objetos y posibilidades de exploración.

Según Picco y Soto (2013), esta trasmisión cotidiana de saberes al bebé constituye un contenido de la enseńanza que puede tener como base aspectos del desarrollo del nińo y de la nińa, la cultura de la crianza y la producción cultural.

Todo desarrollo curricular se verifica en diferentes niveles de concreción. En el centro educativo, se define el proyecto curricular a partir de lineamientos nacionales y al sello institucional. En sala, el educador de párvulos recoge orientaciones nacionales, así como las propuestas específicas del centro y planifica para nińos y niñas que, a su vez, tienen referentes culturales diversos que proceden de los contextos de crianza más próximos. La propuesta curricular que se genere en este nivel de concreción debiese recoger estos saberes y plasmarlos tanto en su diseño como en su implementación.

El bebé recibe sus primeros cuidados y educación en un contexto familiar donde existen formas de acunarlo, alimentarlo, mudarlo, entre otras; es decir, prácticas de los adultos que le van ofreciendo una serie de significados que le 
permiten ir comprendiendo su entorno. En el centro educativo, en la cotidianeidad, los adultos realizan las mismas actividades, como alimentarlo, mudarlo u otras prácticas necesarias para su cuidado y educación. Si ambos escenarios de crianza, familia y centro educativo, se complementan y se influyen mutuamente, contribuyen a su desarrollo y aprendizaje (Violante, 2008a).

Pensar en los contenidos a incorporar en las propuestas curriculares para los bebés involucra el reconocimiento de distintos tipos de saber. Esta selección debiese incluir formas de transmisión cultural presentes en los hogares, específicamente, lo que se relaciona con las prácticas de crianza.

\subsection{Prácticas de crianza como referente del contenido a transmitir en sala cuna}

Las prácticas de crianza remiten a las acciones que los adultos más cercanos al niño realizan para cuidarlo con el fin de cautelar su crecimiento, desarrollo y aprendizaje (Aguirre, 2000). Dar de comer al bebé, cantarle una canción para que se duerma, leerle un cuento, constituirían parte de estas prácticas. Son, efectivamente, acciones cotidianas dirigidas al nińo y nińa que le van trasmitiendo saberes que son parte de una cultura.

De acuerdo con Violante (2008b), «la crianza es un modo de denominar el proceso educativo formativo propio de los primeros ańos» (p. 28), que no solo se da en el escenario del hogar, sino también en los establecimientos educativos y en otros espacios donde interactúan adultos y niños. Consecuentemente, la responsabilidad de criar a los bebés es compartida por distintas instituciones, que debiesen estar articuladas para favorecer su desarrollo. Al respecto, no se trata de reproducir prácticas sin cuestionar aquellas que pudiesen ser perjudiciales para los bebés, sino de comprender los escenarios en que estas se generan y buscar, en conjunto, mejores contextos para estos.

En cuanto a la enseñanza de contenidos en las salas que educan bebés, no existe un criterio compartido. Según Soto y Violante (2005), la dificultad en el abordaje de contenidos estaría relacionada con la falta de consenso respecto al carácter educativo de este nivel, la comprensión sobre las posibilidades de aprendizaje de los párvulos en sus primeros años de vida, la concepción de desarrollo que se sostiene y la ausencia de desarrollos teóricos sobre los contenidos en esta etapa educativa. Si bien las autoras apelan al término «contenido» para referirse a lo que se enseña en este contexto educativo, mencionan que este podría tener fuentes no tradicionales y distintas a las de otros niveles, como las prácticas cotidianas en el hogar y la cultura que se refleja en estas. 
Los contenidos que se transmiten en el nivel sala cuna se asocian más con el «hacer»(Soto, 2008), pues incluyen saberes y prácticas culturales que se enseñan en situaciones cotidianas. Lo anterior implica rescatar las formas en que se realizan estas prácticas; es decir, "recuperar modos tradicionales de crianza hogareña para enseñar a los más pequeños» (Soto y Vasta, 2008, p. 175). En consecuencia, los centros educativos deberían promover el «encuentro de saberes" (Violante, 2008b, p. 27) entre contextos con el objeto de contribuir a una mejor crianza. Conocer y comprender las prácticas que se propician en cada escenario, pues las acciones de los adultos están influenciadas por sus creencias, favorecen ciertos comportamientos en los niños y niñas. En efecto, la crianza se asocia con una cultura específica, una forma de entender el mundo, $\mathrm{y}$ es precisamente esta la que justifica las acciones de los adultos.

En los contextos formales de enseńanza, se organizan horarios para cuidados, como alimentación, mudas y siesta de los bebés; y otros, para favorecer aprendizajes específicos definidos en las propuestas curriculares. Asimismo, se privilegian actividades dirigidas por el adulto, ya sea con todo el grupo o en pequeños grupos. Considerar las prácticas que las familias tienen para criar al bebé como contenido exige revisar las dinámicas que priman en los espacios educativos formales. Soto y Vasta (2008) sugieren reconocer el valor que tienen en sí mismas las actividades cotidianas en cuanto reflejo de una cultura y, por lo tanto, pensar en lo que aportan al bebé. Una actividad de alimentación puede abordarse desde la convivencia, la inculcación de normas, el intercambio verbal, la adquisición de mayor autonomía, entre otras.

La construcción de propuestas curriculares comienza por la selección de lo que se va a enseñar, implica reflexionar sobre el tipo de saber que será trasmitido en el contexto escolar. El intercambio de saberes en torno a la crianza, entre la sala cuna y las familias que envían a sus hijos a este nivel educativo, debiese ser el comienzo de la elaboración de estas propuestas, en cuanto refleja las miradas que se tiene en cada uno de los contextos con respecto a la educación, desarrollo y aprendizaje de los bebés.

\section{Metodología}

La investigación desarrollada se abordó bajo un enfoque cualitativo, puesto que se quería "comprender el comportamiento humano desde el marco de sus protagonistas» $\mathrm{y}$ «observar el fenómeno en su contexto» (Vieytes, 2004, p. 72). Además, tuvo un alcance exploratorio y descriptivo (Hernández, Fernández y Baptista, 2010). 
Correspondió a un estudio de caso, "una investigación exhaustiva y desde múltiples perspectivas de la complejidad y unicidad de un determinado proyecto, política, institución, programa o sistema en un contexto real» (Simons, 2011, p. 42). Específicamente, interesaba explorar y describir prácticas de crianza e intercambios en torno a estas entre un contexto educativo y los hogares de los niños y niñas que asistían a sala cuna. Se trató de un estudio de caso múltiple que incluyó dos centros educativos de educación parvularia de distinta dependencia administrativa y ubicación geográfica, y, en este apartado, se presenta un solo caso de estudio (Bravo, 2018).

El trabajo de recolección de datos estuvo a cargo de la investigadora principal y se prolongó por más de tres meses. Las visitas se coordinaron con anticipación y su frecuencia fue de uno o dos días por semana, durante media o toda la jornada, dependiendo de los datos a recabar. La primera etapa contempló el análisis de documentos del jardín infantil, su proyecto educativo y las planificaciones del nivel sala cuna, con el propósito de conocer el sello del establecimiento, principios y valores en los cuales se enmarca el proceso educativo, además de las actividades que se planifican para los niños y niñas en el aula y las orientaciones para el trabajo con la familia y comunidad. Se dejó un registro escrito de esta revisión. Antes de realizar las entrevistas, se hizo una primera observación panorámica - no participante (Ruiz, 2003) de toda la jornada diaria (8:30 a 17:00). Se observaron y registraron por escrito las actividades que realizaban los niños y niñas, las acciones de los adultos y sus interacciones con los párvulos, además de las características del espacio y materiales. Con respecto a los hogares de los bebés, no fue posible realizar observaciones.

En una segunda etapa, se consideró entrevistas en profundidad (Ruiz, 2003) al personal del establecimiento. Se trabajó con un guion de preguntas abiertas relacionadas con las concepciones sobre la crianza y la descripción de las dinámicas diarias en la sala cuna (interacciones con los niños y niñas, actividades que estos realizan y el uso de los espacios y materiales), así como con los intercambios que tienen con las familias acerca de la crianza (frecuencia, modalidad, temas que se abordan). De acuerdo con el curso que iba tomando la conversación, se incorporaron otras preguntas para profundizar o ampliar el contenido de las mismas. Estas conversaciones se extendieron entre 60 y 80 minutos. Se entrevistó a la coordinadora pedagógica del ciclo, a la trabajadora social, a la educadora de párvulos de la sala cuna menor y a las dos técnicos del nivel, quienes fueron seleccionadas por estar en contacto más directo con los bebés y sus familias. Estas entrevistas fueron grabadas previo consentimiento informado. Una vez finalizado este ciclo de entrevistas, se realizó una segunda 
observación de la jornada diaria en la sala cuna para profundizar en la dinámica diaria de la sala cuna.

Por último, se llevaron a cabo las entrevistas a los apoderados de la sala cuna. El guión incluía preguntas abiertas sobre sus concepciones sobre la crianza y las dinámicas diarias en sus hogares con respecto a las interacciones con sus hijos e hijas, actividades que estos realizan, los espacios y materiales que utilizan, además de los intercambios que tienen con el personal del establecimiento educativo. Se entrevistó a todos aquellos que tenían disponibilidad horaria y que accedieron a ser entrevistados, once madres de un total de dieciocho. No se presentó ningún padre a las entrevistas. Todas las entrevistas fueron grabadas previo consentimiento informado.

Con respecto al análisis de los datos cualitativos (Gibbs, 2012), se transcribieron las entrevistas y se digitaron los registros de las observaciones y del análisis de los documentos. Este material se leyó acuciosamente en varias oportunidades y se fue organizando en categorías y subcategorías a las que se les asignaron códigos. Inicialmente, las categorías estaban preconcebidas, pero producto del análisis de los datos, estas se fueron modificando y precisando. La síntesis de los datos se expuso en matrices. La descripción de las prácticas de crianza que prevalecen en el contexto sala cuna, emerge de una "triangulación de datos» (Denzin, 1978 citado en Simons, 2011 p. 182) de los documentos analizados, las entrevistas al personal y las observaciones en sala. Con respecto a la descripción de las prácticas que prevalecen en el hogar, estas surgen a partir de las tendencias encontradas tras el análisis de las entrevistas realizadas a las madres.

\section{Resultados}

El caso de estudio correspondió a una sala cuna menor de un jardín infantil de una comuna urbana con más de treinta años de funcionamiento, perteneciente a una congregación religiosa de gestión privada. La sala cuna atendía a dieciocho niños y niñas, entre 5 y 14 meses, a cargo de una profesional educadora de párvulos y de dos técnicos en párvulos. Los bebés permanecían en el centro gran parte del día, de lunes a viernes, desde las 8.30 a las 17.00 hrs. Las familias cuyos hijos e hijas asistían al nivel, en su mayoría, vivían cerca del establecimiento, se desempeñaban en diferentes rubros, principalmente, en el área de servicios y en trabajos informales y pertenecían a los quintiles de menos ingresos socioeconómicos. 


\subsection{Prácticas de crianza en la sala cuna descritas a partir de las observaciones, entrevistas y análisis de documentos}

El personal entrevistado entiende por prácticas de crianza, principalmente, a las acciones que realiza la familia cotidianamente para guiar y educar al niño y niña. Las prácticas juzgadas como apropiadas son aquellas que se orientan por un buen trato hacia los párvulos. Especialmente, se valora la preocupación por su desarrollo y la interacción que se tenga con él o ella. Mientras que las juzgadas como inapropiadas corresponden a aquellas que atentan o vulneran algún derecho como negligencia con respecto a su higiene, maltrato físico, mucha permisividad o exceso de autoritarismo.

Con respecto a las interacciones de los adultos con los bebés, estas se dan en un clima de afecto y calidez, se promueve el buen trato, lo que implica la creación de lazos afectivos y de respeto por el párvulo como sujeto de derechos. Así también, se visualiza una intencionalidad pedagógica en las interacciones diarias. En ciertos periodos de la jornada, un adulto preferentemente es quien dirige las actividades de todos los niños y niñas, apoyado por los otros adultos de la sala. En otros momentos, se trabaja en subgrupos y cada adulto se dirige solo a un pequeño grupo de bebés. Las interacciones incluyen verbalizaciones y expresiones gestuales relacionadas con la marcha de la rutina diaria.

Los bebés se incorporan a juegos y actividades organizados con una finalidad pedagógica, divididos en subgrupos según edad. Estas actividades han sido planificadas para favorecer distintos ámbitos de experiencias, como formación personal y social, comunicación y relación con el medio natural y cultural, privilegiándose las sensoriales, motricidad y lenguaje verbal. El adulto dirige la actividad, agrupa a los bebés, les da indicaciones y, después, se realizan las acciones propuestas. Se utilizan señales para marcar la finalización de una actividad. Los bebés autoinician sus actividades solo en periodos de transición, cuando esperan un cambio de actividad. La mayoría de las actividades organizadas implica el uso de algún tipo de material concreto, y se incluyen, además, cantos y oraciones vinculados al sello institucional (congregación religiosa).

En relación con los periodos de higiene y mudas, se observa que los bebés son revisados periódicamente y se les muda en distintos periodos de la jornada acorde a sus requerimientos. Se les ubica en un mudador dentro del baño, se resguardan las condiciones de higiene y el clima en que se desarrolla. Se aprovecha esta instancia para una interacción más individualizada con el bebé, se intenciona su lenguaje verbal y gestual y se fortalece el vínculo con este. Se incluye, además, la higiene de manos y cara, el cepillado dental, el peinado y uso de servilleta, y hábitos de higiene ambiental, como el uso de papeleros. 
Los periodos de alimentación se realizan todos los días en un mismo horario e incluyen: desayuno, leche, alrededor de las 9:30; almuerzo, alrededor de las 11:30 y una leche a las 15:00. Se ambienta la sala incorporando una mesa y una banca para los bebés que ya caminan, el resto se ubica en sillas de comer y se les coloca un babero. Los adultos se organizan para alimentarlos y se cautela que la alimentación se dé dentro de un clima grato. Se utilizan distintos recursos, como cantos y juegos, para ayudar al bebé a recibir los alimentos, pero no se le obliga a ingerirlos si no tiene hambre. Las preparaciones incluyen variados tipos de alimento, como carne de ave y de vacuno, legumbres, cereales, frutas y verduras. Las preparaciones varían de acuerdo con la edad del bebé. Se fomenta la lactancia materna, por ello, las madres asisten durante la jornada a amamantar a sus hijos e hijas.

Con respecto a los periodos de siesta, estos se realizan diariamente después del almuerzo. En el caso de los más pequeños, si se quedan dormidos en otros periodos, son ubicados en sus cunas. Se ambienta la sala, se oscurece, se pone música suave y cada bebé es ubicado en su cuna. Se les saca un poco de ropa para mayor comodidad. Algunos son ayudados con unas caricias en su cabeza o con unos golpecitos en la espalda. Siempre quedan dos adultos supervisando la siesta de los bebés.

Las normas y la disciplina se promueven diariamente dentro de un contexto valórico, donde prima el buen trato como parte del sello institucional. Se corrige al bebé en un tono afectuoso y fraterno y se enfatiza la convivencia grupal.

En relación con el espacio y materiales, todo está organizado con una finalidad pedagógica, considerando los requerimientos de las edades de los bebés. La sala es amplia y tiene áreas implementadas con repisas y objetos al alcance del bebé, aunque de preferencia estos son utilizados cuando el adulto se lo permite. Existe abundante material variado en tamaño, textura, color y forma, como legos, encajes, figuras de animales, muńecos y libros. Además, se cuenta con una sala para el trabajo de psicomotricidad donde se incluyen implementos y materiales como colchonetas, barras, rampas y pelotas. La decoración es alusiva al tema que se está trabajando. Se utilizan, preferentemente, la sala y el hall interior, y, cuando el clima lo permite, un patio exterior. El uso de los espacios es más libre en los periodos de transición.

\subsection{Prácticas de crianza en el hogar descritas por las madres}

Las madres opinan que las prácticas de crianza constituyen cualquier actividad que se vincule con «criar al nińo y niña». Juzgan como apropiado «educar al 
bebé con afecto». Mientras que consideran inapropiado todo lo que involucra «maltratarlos física o verbalmente».

Con respecto a las interacciones entre los adultos y los bebés, las entrevistadas declaran que estas se caracterizan por un fuerte tono afectivo con muchas expresiones de afecto, tanto gestuales, como verbales dirigidas hacia sus hijos e hijas. Asimismo, mencionan que muchas veces intencionan aprendizajes, por ejemplo, repiten palabras para que el bebé desarrolle su lenguaje o realizan acciones para que las imite.

En relación con los juegos y actividades, mencionan que incluyen a sus bebés en aquellas actividades que desarrollan habitualmente en el hogar, como guardar y ordenar utensilios; asimismo, loa incluyen en las rutinas familiares, como sentarse en la mesa a la hora de las comidas o ir de compras, y también en aquellas actividades que los adultos de la casa disfrutan, como cantar y bailar. Igualmente, señalan que algunas organizan actividades específicas para que los bebés aprendan y se entretengan, como jugar con objetos, cantar y escuchar cuentos.

Con respecto a los periodos de higiene y mudas, las madres cuentan que revisan periódicamente al bebé, lo mudan según requerimientos, sin que haya un horario establecido para ello. Se preocupan, además, de propiciar un periodo grato y de intercambio, permitiendo al bebé que juegue y explore. Generalmente, mudan en el dormitorio. Incluyen otras actividades de higiene, como lavado de manos y cara, cepillado dental y baño completo.

En cuanto a los periodos de alimentación, mencionan que utilizan una silla de bebé para alimentarlo. Generalmente, lo hacen en un mismo horario e intentan seguir siempre una misma rutina. Las preparaciones varían, molida para los más pequeños y semisólida para los más grandes; se incorporan frutas, verduras, vacuno, pollo, cereales y lácteos. Si el bebé no recibe los alimentos, utilizan estrategias como el canto, los gestos y movimientos para distraerlo. Muchas declaran que todavía amamantan a sus bebés y que no permiten la ingesta de comida chatarra a sus hijos e hijas.

Respecto a la siesta, indican que los bebés duermen de acuerdo con sus requerimientos, generalmente, después de ser alimentados y su extensión es variable. Agregan que estos tienen un espacio definido para dormir y que utilizan distintas estrategias para ayudarlos a conciliar el sueńo, por ejemplo, ubicarlos boca abajo, pasarle un objeto conocido «tuto", acunarlos, cantarles, y darle pequeños golpecitos en la espalda.

Declaran que inculcan normas y disciplina al bebé enseñándole a discernir entre lo que es apropiado y lo que no es. Utilizan estrategias verbales para advertirle las consecuencias de sus acciones y evitar el castigo. Lo premian para 
estimular su buen comportamiento y suprimen algo de su agrado o lo dejan en su dormitorio como castigo.

En relación con el uso de los espacios y materiales, explican que adaptan espacios interiores en sus departamentos y que, en general, sus bebés cuentan con objetos y juguetes apropiados a la edad, los que pueden utilizar en cualquier momento del día. Salen frecuentemente con sus bebés, los llevan al supermercado, al centro de la ciudad, a plazas y áreas verdes cercanas al sector.

\subsection{Intercambios en torno a las prácticas de crianza entre las familias y el jardín infantil}

Con respecto al rol del jardín infantil, el personal de la sala cuna destaca su rol pedagógico y los énfasis del proyecto educativo: buen trato, enfoque de género, alimentación saludable y valores cristianos. Asimismo, reconoce el apoyo que el centro le brinda a la familia. Respecto del rol de la familia, reconoce a esta como la primera educadora de los párvulos, que comparte esta tarea con el jardín infantil. Valora la preocupación que las familias demuestran por la educación de sus bebés, las describe como "participativas e involucradas en la educación de sus hijos». Sin embargo, la intención de rescatar ese saber es muy incipiente.

Por otra parte, las madres mencionan que el rol del jardín infantil es asistencial y pedagógico. Con respecto al rol de las familias, lo fundamental para las entrevistadas sería "criar y educar al bebé, entregándole una base sólida», así como apoyar la labor del centro.

El jardín infantil tiene instancias formales para intercambiar con las familias, estas incluyen entrevistas, reuniones periódicas, talleres y charlas relacionadas con las prioridades del proyecto educativo. También, organiza actividades culturales y recreativas para las familias. Se aprecian, por otra parte, instancias menos formales, especialmente, en los horarios de ingreso o de salida, donde un adulto de la sala conversa en forma más individualizada con un familiar del bebé. Se mantienen registros escritos para eventualidades, por ejemplo, cuando un bebé se retira más temprano porque se enfermó. Se observan registros de talleres sobre alimentación saludable y salud del párvulo, realizados con apoyo del Centro de Salud Familiar (Cesfam) del sector. El personal declara que todas las instancias de encuentro tienen como principal propósito crear lazos de confianza con las familias y que valora la excelente disposición que estas tienen para acoger lo que el jardín infantil les brinda como apoyo a la educación de sus hijos e hijas. 
Respecto del contenido del intercambio en torno a la crianza de los bebés, el personal indaga al inicio del año escolar sobre los contextos hogareños, como la composición familiar, las condiciones de la vivienda, las actividades y los estudios formales de los padres y madres. Pero, escasamente, saberes y prácticas de crianza. Los intercambios referidos a los horarios del bebé, sus hábitos de sueńo, ingesta de alimentos y las mudas, se producen en el contacto habitual con el adulto que lo deja y/o retira del establecimiento.

\subsubsection{Intercambio de saberes en torno a la crianza y su inclusión en la propuesta curricular}

Se aprecia que los documentos institucionales especifican orientaciones para la implementación curricular relacionadas con los énfasis de las interacciones con los párvulos, como el buen trato, la promoción de una educación no sexista y un estilo de vida saludable, basados en la perspectiva de los Derechos del Niño. Se han establecido, además, protocolos para el trabajo en el aula donde se indica lo que es apropiado para la higiene del bebé y los casos en que se debe denunciar cualquier situación de negligencia en este ámbito. Sin embargo, no se aprecia el aporte que pudiesen realizar las familias con respecto a estas prácticas de crianza, como recoger lo que estas entienden por buen trato, en qué sustentan esta percepción, lo mismo en cuanto a las prácticas de higiene y las condiciones que tienen para ello en sus hogares.

De acuerdo con lo declarado por el personal, se valoran las prácticas de las familias y la idea de una continuidad en las prácticas de crianza entre el hogar y el centro. No obstante, existe la creencia que principalmente es la familia la que aprende del centro educativo, pues es este contexto quien puede realizar aportes, entregándoles orientaciones sobre la educación del bebé. Por otra parte, las familias confían mucho en el saber que proviene desde el jardín infantil.

Se aprecia que la práctica de mudar al bebé en sala es similar a la del hogar. Si bien es una práctica habitual en el centro, no se observa una reflexión en el sentido de recuperar otras prácticas hogareñas, a pesar de que el personal reconoce que habría que lograr un mayor intercambio de saberes en torno a la crianza de los bebés con el propósito de incorporarlos en las propuestas del jardín infantil. Del mismo modo, algunas de las madres señalan que pueden hacer aportes relacionados con la crianza de sus hijos e hijas y destacan que en ambos contextos se enseña a los bebés. 


\section{DisCUSIÓN Y CONCLUSIONES}

Se observa que tanto en la sala cuna como en el contexto hogareño se enfatiza un buen trato hacia los bebés. En sala cuna, las prácticas de los adultos se enmarcan en una perspectiva de "Derechos del Nińo» y se sanciona toda acción que implique su vulneración. Por otra parte, las madres entrevistadas destacan la importancia del contacto físico y afectividad con los niños y niñas y el rechazo a prácticas que implican agresión física o verbal hacia los bebés. En cuanto a los juegos y actividades en las que participan los bebés, en sala cuna se planifican y estructuran en función de los aprendizajes que se quieren favorecer. Mientras que, en el contexto hogareño, las madres opinan que ellas intencionan aprendizajes a través de las interacciones y acciones que realizan cotidianamente con sus hijos e hijas. En cuanto a las prácticas de cuidado, en sala se definen horarios para los periodos de alimentación y siesta, mientras que las mudas son flexibles. De acuerdo con lo declarado por las madres, no hay horarios definidos para estos periodos, no obstante, se repiten rutinas.

En cuanto al intercambio de saberes en torno a estas prácticas y su inclusión en la propuesta curricular del centro, se aprecian elementos incipientes. Se constata que los documentos institucionales estipulan orientaciones específicas sobre prácticas de buen trato, visibles en el discurso del personal y en algunas prácticas en sala. Asimismo, se observa que el personal de la sala cuna se comunica diariamente con las familias en torno a los periodos de alimentación, higiene y descanso y al comportamiento del bebé. Sin embargo, no se observan instancias de reflexión en cuanto al sentido de estos intercambios y cómo aprovecharlos en la construcción de propuestas curriculares.

En el contexto de la ampliación de la cobertura de atención en el nivel sala cuna, pensar la educación de los bebés y las propuestas curriculares que se generen constituye una prioridad. Los hallazgos de este estudio nos señalan algunos temas a discutir.

En ambos contextos, alimentarse, jugar con objetos, dormir, ser mudado, son actividades habituales de los bebés, que tienen la particularidad de ser acompañadas por la presencia de adultos, quienes a través de la forma en que realizan las acciones van trasmitiendo saberes inscritos dentro de un referente cultural específico. Al respecto, los espacios educativos formales debiesen rescatar las prácticas que los adultos tienen hacia los bebés en los contextos hogareños e incorporarlas como referentes para el "qué se enseña» en este nivel. No es suficiente declarar la relevancia de los contextos en que se crían los bebés, sino dar sentido a los intercambios que se generan. En la misma línea de lo señalado por Soto y Violante (2005), observamos que la idea de 
contenido en este ciclo educativo resulta compleja, se tiende a sobrevalorar lo que se prescribe en los currículos oficiales y, por lo tanto, su definición se deja a los expertos, desconociéndose el aporte que pueden realizar otros actores, especialmente, las familias de los bebés.

Por otra parte, las distintas formas de instalar aprendizajes en ambos contextos sugieren revisar las prácticas en la sala cuna y ampliar los referentes que se tienen con respecto al "cómo se enseña». Considerar los saberes y experiencias que tienen las familias para criar y educar a sus hijos e hijas, involucra que en los espacios educativos formales se aprovechen instancias y acciones habituales para intencionar aprendizajes.

Pensar en "qué enseñar a los bebés» y en "cómo hacerlo», requiere una revisión profunda del proceso de construcción curricular y, en este sentido, la forma y tipo de intercambio que se genere entre ambos escenarios, hogar y centro educativo, puede facilitar o dificultar la tarea. Consideramos que los centros educativos que educan bebés demandan otras miradas con respecto a lo que se valida como saber, pues solo de esta forma se podrán realizar procesos de construcción curricular más pertinentes, dando espacio a otros actores, que no necesariamente cuentan con estudios especializados en el área de la infancia, pero sí poseen saberes acerca de la crianza y educación de niños y niñas.

La crianza de nińos y niñas ha sido influenciada, permanentemente, por las transformaciones sociales y culturales que se han producido a través de los años. Por lo tanto, resulta lógico recuperar saberes acerca de la crianza provenientes de las familias y comunidades donde se desarrolla la acción educativa. Lo anterior implica considerar, como uno de los referentes del contenido curricular y de la modalidad de enseñanza en sala cuna, a las prácticas de crianza de los hogares, es decir, a las creencias, costumbres, valores, actitudes y pautas de comportamiento que se transmiten a los niños y niñas cotidianamente. Seleccionar conjuntamente lo que se transmitirá en el contexto educativo comporta que las familias conozcan y comprendan lo que se pretende enseñar en estos contextos y sus referentes y, a la vez, requiere de educadores y educadoras dispuestos a transformar los contextos educativos formales. En virtud de ello, la construcción de propuestas curriculares debiese comenzar por intercambiar y confrontar las distintas posiciones con respecto al cuidado y educación de los niños y niñas y los referentes que se tienen para ello.

Por último, se señala que el no haber realizado observaciones en los contextos hogareńos pudo haber sido una limitante del estudio. Se sugiere profundizar en este tipo de estudio, especialmente, en lo vinculado con la selección de contenidos educativos para los bebés. 


\section{REFERENCIAS BIBLIOGRÁFICAS}

Aguirre, E. (2000). Socialización y Prácticas de crianza. En E. Aguirre y E. Durán, Socialización: prácticas de crianza y cuidado de la salud. Un estudio con familias y niños que inician su escolarización en Santa Fe de Bogotá. Colombia: Centro de Estudios Sociales. Universidad Nacional de Colombia. Recuperado de http: //www.bdigital.unal.edu.co/15487/5/02CAPI01.pdf

Bravo-Soto, A. (2018). Knowledge about Child Rearing Practices: Exchange Between Nursery and Family. Revista Electrónica Educare, 22(3), 1-25. https://doi.org/10.15359/ree.22-3.5

Hernández, R., Fernández, C. y Baptista, P. (2010). Metodología de la investigación. Quinta edición. México: Mc Graw-Hill.

Gibbs, G. (2012). El análisis de datos cualitativos. Madrid: Morata.

Marotta, E. (2009). La didáctica del jardín entendida como construcción colectiva. En E. Marotta, M. Rebagliati y C. Sena (Coords.), Jardín infantil o escuela maternal. Ecos de una experiencia docente (pp. 77- 86). Buenos Aires: Novedades Educativas.

Ministerio de Desarrollo Social. (2016). Encuesta de Caracterización Socioeconómica Casen, 2015. Recuperado de http://observatorio.ministeriodesarrollosocial.gob.cl

Ministerio de Educación. (2005). Bases Curriculares de la Educación Parvularia. Santiago de Chile: Maval.

Picco, P. y Soto, C. (2013). Construyendo experiencias de enseńanza y cuidado en las instituciones para la primera infancia. Temas para niños y nińas menores de tres ańos. Documento. Recuperado de http:/www.des.for.infd. edu.ar/sitio/upload/Que_y_como_ensenar_a_ninos_pequenos_1.docx

Pitluk, L. (2011). Educar en el jardín maternal: enseñar y aprender de 0 a 3 años. (2a reimp.). Buenos Aires: Novedades Educativas.

Ramírez, P. (2009). La problemática del conocimiento en el jardín maternal. En E. Marotta, M. Rebagliati y C. Sena (Coords.), Jardin infantil o escuela maternal. Ecos de una experiencia docente (pp. 69-76). Buenos Aires: Novedades Educativas.

Ruiz, J. (2003). Metodología de la Investigación Cualitativa. (3a ed.). España: Universidad de Deusto.

Simons, H. (2011). El estudio de caso: Teoría y práctica. Cap. VII. Madrid: Morata.

Soto, C. (2008). Distintas voces hablan de la enseñanza. En C. Soto y R. Violante, Pedagogía de la crianza: un campo teórico en construcción (pp. 155-171). Buenos Aires: Paidós. 
Soto, C. y Vasta, L. (2008). Del alfabeto cultural: prácticas cotidianas y juegos tradicionales. En C. Soto, C. y R. Violante, Pedagogía de la crianza: un campo teórico en construcción (pp. 173-195). Buenos Aires: Paidós.

Soto, C. y Violante, R. (2005). Enseñar contenidos en el jardín maternal: una forma de compartir la crianza. En C. Soto, C. y R. Violante (Comps.), En el jardin maternal, investigaciones reflexiones y propuestas (pp. 31- 65). Buenos Aires: Paidós.

Vieytes, R. (2004). Metodología de la Investigación en organizaciones, mercado y sociedad. Buenos Aires: De las ciencias.

Violante, R. (2008a). Los escenarios de crianza en la educación de los niños pequeños. En C. Soto, C. y R. Violante, Pedagogía de la crianza: un campo teórico en construcción (pp. 55-75). Buenos Aires: Paidós.

Violante, R. (2008b). Por qué pedagogía de la crianza. En C. Soto, C. y R. Violante, Pedagogía de la crianza: un campo teórico en construcción (pp. 23-53). Buenos Aires: Paidós. 